\begin{tabular}{|c|l|}
\hline Title & Robust Existence of a Reaction Boundary to Separate the Fate of a Chemical Reaction \\
\hline Author(s) & Kawai, Shinnosuke; Komatsuzaki, Tamiki \\
\hline Citation & $\begin{array}{l}\text { Physical Review Letters, 105(4), 048304 } \\
\text { https://doi.org/40.1103/PhysRevLett.105.048304 }\end{array}$ \\
\hline Issue Date & 2010-07-23 \\
\hline Doc URL & http://hdl.handle.net/2115/43651 \\
\hline Rights & ○ 2010 The A American Physical Society \\
\hline Type & article \\
\hline File Information & PRL105-4_048304.pdf \\
\hline
\end{tabular}

Instructions for use 


\title{
Robust Existence of a Reaction Boundary to Separate the Fate of a Chemical Reaction
}

\author{
Shinnosuke Kawai ${ }^{1, *}$ and Tamiki Komatsuzaki ${ }^{1,2}$ \\ ${ }^{1}$ Molecule and Life Nonlinear Sciences Laboratory, Research Institute for Electronic Science, Hokkaido University, \\ Kita 20 Nishi 10, Kita-ku, Sapporo 001-0020, Japan \\ ${ }^{2}$ Core Research for Evolutional Science and Technology (CREST), Japan Science and Technology Agency (JST), \\ Kawaguchi, Saitama 332-0012, Japan
}

(Received 15 January 2010; revised manuscript received 2 March 2010; published 21 July 2010)

\begin{abstract}
Nonlinear dynamics around a rank-one saddle is investigated in a high energy regime above the reaction threshold. The transition state (TS) is considered as a surface of a "point of no return" through which all reactive trajectories pass only once in the process of climbing over the saddle before being captured in the product state. A no-return TS ceases to exist above a certain high energy regime. However, even at high energies where the no-return TS can no longer exist, it is shown that "an impenetrable barrier" in the phase space robustly persists, which acts as a boundary between reactive and nonreactive trajectories. This implies that we can yet predict the fate of reactions even when the no-return TS may not exist. As an example, we show the analysis of dynamical systems theory for a hydrogen atom in crossed electric and magnetic fields.
\end{abstract}

DOI: 10.1103/PhysRevLett.105.048304

The dynamics around rank-one saddles plays a crucial role in the study of chemical reactions. This is because in most chemical reactions there exists a saddle point between one stable structure (reactant) and another (product), functioning as a "barrier" for the reaction. The occurrence of the reaction is primarily determined by the possibility of surmounting this barrier. Studies of the dynamics in the vicinity of the saddle have made great contributions to the calculation of reaction rates, as well as to physical insights into not only reaction dynamics [1-6] but also, for example, ionization of a hydrogen atom in crossed electric and magnetic fields [7,8], isomerization of clusters [9], the escape of asteroids from Mars [10], the diffusion of impurities in crystalline materials [11], and the folding or unfolding of proteins $[12,13]$. Among the central concepts in the study of dynamics in the saddle region are the "transition state" (TS) [14-21] and several invariant manifolds $[22,23]$. The TS is originally defined as a surface dividing the phase space into two distinct regions, i.e., reactant and product, through which the system passes once and only once when undergoing the reaction from one region before being "captured" in the other region. In other words, the TS separates the space into "before" and "after" the reaction. In turn, an invariant manifold is a set of points in the phase space such that, once the system is in that manifold, the system will stay in it perpetually. As a consequence, no trajectory in the phase space can cross the invariant manifold whose dimension is less than that of the space by one from one side to the other. One of the important invariant manifolds for understanding the reactions is that which separates reactive trajectories from nonreactive ones, so that once we know which side of the manifold a given initial condition is, we can immediately know, without any trajectory calculations, whether the initial condition brings the system to the product or not.
PACS numbers: 82.40.Bj, 05.45.-a, 82.20.Db, 82.40.Ck

If the total energy of the system is only slightly above the saddle point, the Hamiltonian can be well approximated by

$$
\begin{aligned}
H(\boldsymbol{q}, \boldsymbol{p}) & =\frac{p_{1}^{2}}{2}-\frac{\lambda^{2}}{2} q_{1}^{2}+\sum_{l=2}^{n}\left(\frac{p_{l}^{2}}{2}+\frac{\omega_{l}^{2}}{2} q_{l}^{2}\right) \\
& =\lambda x \xi+\sum_{l=2}^{n}\left(\frac{p_{l}^{2}}{2}+\frac{\omega_{l}^{2}}{2} q_{l}^{2}\right),
\end{aligned}
$$

with the so-called normal mode coordinates $\left(q_{1}, \ldots, q_{n}\right)$, which diagonalize the potential energy, and their conjugate momenta $\left(p_{1}, \ldots, p_{n}\right)$. Here we assign the unstable direction as mode 1 , with the curvature of the potential $-\lambda^{2}$. The other modes are vibrational modes with frequencies $\omega_{l}$. We have introduced the following variables,

$$
x \stackrel{\text { def }}{=} \frac{\lambda q_{1}+p_{1}}{\sqrt{2 \lambda}}, \quad \xi \stackrel{\text { def }}{=} \frac{p_{1}-\lambda q_{1}}{\sqrt{2 \lambda}},
$$

which define a skewed coordinate system $(x, \xi)$. The action variable defined by $I \stackrel{\text { def }}{=} x \xi$ is an invariant of motion with the Hamiltonian given by Eq. (1). Therefore the trajectories run along the hyperbolas given by $I=$ const as shown in Fig. 1(a).

Suppose that $q=-\infty$ corresponds to the "reactant" (i.e., before the reaction), and $q=+\infty$ to the "product" (after the reaction). The trajectories with $x>0$ and $\xi>0$ are "forward reactive" because they start from the reactant, overcome the barrier, and go into the product (i.e., the reaction occurs from the reactant to the product). On the other hand, the trajectories with $x<0$ and $\xi>0$ are "forward nonreactive" because they start from the reactant, but are reflected by the barrier, and go back into the reactant. Similarly, the trajectories with $x<0$ and $\xi<0$ are "backward reactive," and those with $x>0$ and $\xi<0$ are "back- 

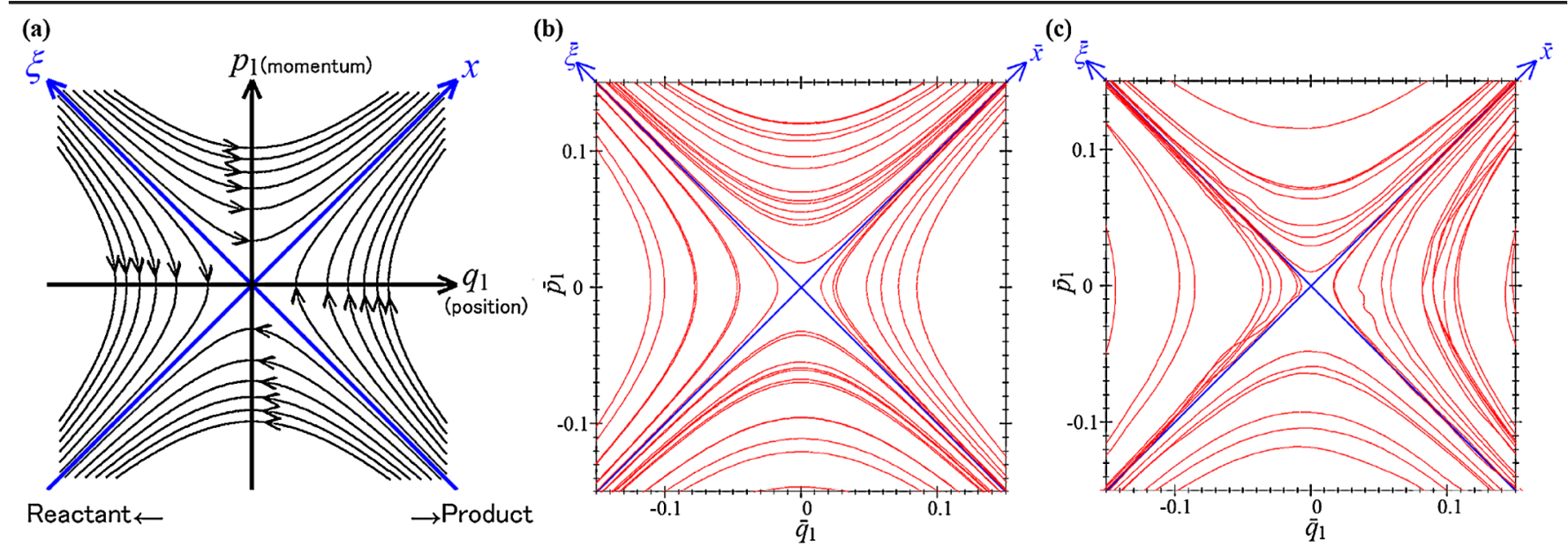

FIG. 1 (color online). Phase space flows in the saddle region. (a) Flow for the case of harmonic approximation. (b) Representative trajectories of the hydrogen atom in crossed electric and magnetic fields, with energy $E=0.05$, projected on the partial normal form coordinates. (c) Those with $E=0.15$, projected on the minimal normal form coordinates.

ward nonreactive." Thus the sets $\{x=0\}$ and $\{\xi=0\}$ constitute the boundaries (=invariant manifolds) between the reactive and the nonreactive trajectories.

As the total energy of the system increases, the harmonic approximation Eq. (1) becomes no longer valid. We have higher order terms in the Taylor expansion of the Hamiltonian:

$$
\begin{aligned}
H(\boldsymbol{q}, \boldsymbol{p})= & \lambda x \xi+\sum_{l=2}^{n}\left(\frac{p_{l}^{2}}{2}+\frac{\omega_{l}^{2}}{2} q_{l}^{2}\right) \\
& +H^{\prime}\left(x, \xi, q_{2}, \ldots, q_{n}, p_{2}, \ldots, p_{n}\right),
\end{aligned}
$$

where $H^{\prime}$ is a polynomial function containing cubic and higher order terms. While $H^{\prime}$ can take any kind of functional form, recent studies [7-9,22-27] have found that it is possible to introduce a coordinate transformation $\quad\left(x, \xi, q_{2}, \ldots, q_{n}, p_{2}, \ldots, p_{n}\right) \mapsto(\bar{x}, \bar{\xi}$, $\left.\bar{q}_{2}, \ldots, \bar{q}_{n}, \bar{p}_{2}, \ldots, \bar{p}_{n}\right)$ that casts the Hamiltonian into the following form:

$$
\begin{aligned}
H(\boldsymbol{q}, \boldsymbol{p}) \approx & \bar{H}\left(\bar{x}, \bar{\xi}, \bar{q}_{2}, \ldots, \bar{q}_{n}, \bar{p}_{2}, \ldots, \bar{p}_{n}\right) \\
= & \lambda \bar{x} \bar{\xi}+\sum_{l=2}^{n}\left(\frac{\bar{p}_{l}^{2}}{2}+\frac{\omega_{l}^{2}}{2} \bar{q}_{l}^{2}\right) \\
& +f_{1}\left(\bar{I}, \bar{q}_{2}, \ldots, \bar{q}_{n}, \bar{p}_{2}, \ldots, \bar{p}_{n}\right) \\
& +f_{2}\left(\bar{q}_{2}, \ldots, \bar{q}_{n}, \bar{p}_{2}, \ldots, \bar{p}_{n}\right) ;
\end{aligned}
$$

that is, the total Hamiltonian depends on $\bar{x}$ and $\bar{\xi}$ only through the action $\bar{I} \stackrel{\text { def }}{=} \bar{x} \bar{\xi}$. Here $f_{1}$ and $f_{2}$ contain anharmonic terms, and in particular, $f_{1}$ is defined so that $f_{1}=0$ when $\bar{I}=0$. It is easily seen that $\bar{I}$ is an invariant of motion, so that we can have the same picture as Fig. 1(a) just by replacing the names of the axes with $\bar{x}$ and $\bar{\xi}$. The transformation is constructed by Lie canonical perturbation theory $[28,29]$ (a classical analog of Van Vleck perturbation theory [26]). The form of Eq. (4) is called "partial normal form" (PNF) in the sense that only the action of the reactive mode is transformed as an invariant, which survives robustly even at a moderately high energy regime (because resonance does not meet between the reactive and nonreactive modes [7-9,22-27]). It is also possible to construct a "full normal form" that makes all the actions transform as invariants of motion [7-10,22-25]. Since the normal form transformation is based on the perturbation theory, one must be careful about its convergence. In many cases the perturbation series suffer from divergence mainly arising from the appearance of nonlinear resonances among the modes when the system has significant nonlinear couplings at a high energy regime. In general, the convergence improves when the transformation makes lesser action variables into invariants of motion because the transformation is then closer to the identity transformation.

In this Letter, we suggest another form of normal form

$$
\begin{aligned}
\bar{H}\left(\bar{x}, \bar{\xi}, \bar{q}_{2}, \ldots, \bar{q}_{n}, \bar{p}_{2}, \ldots, \bar{p}_{n}\right) \\
=\lambda \bar{x} \bar{\xi}+\sum_{l=2}^{n}\left(\frac{\bar{p}_{l}^{2}}{2}+\frac{\omega_{l}^{2}}{2} \bar{q}_{l}^{2}\right) \\
\quad+\bar{I} g_{1}\left(\bar{x}, \bar{\xi}, \bar{q}_{2}, \ldots, \bar{q}_{n}, \bar{p}_{2}, \ldots, \bar{p}_{n}\right) \\
\quad+g_{2}\left(\bar{q}_{2}, \ldots, \bar{q}_{n}, \bar{p}_{2}, \ldots, \bar{p}_{n}\right) .
\end{aligned}
$$

This functional form is closer to the original Hamiltonian than the other normal forms in that it allows for any form of $g_{1}$ and $g_{2}$, but $g_{1}$ has a prefactor $\bar{I}$. It is therefore expected to have a better convergence property. Under the Hamiltonian given by Eq. (5), the action variable $\bar{I}$ is no longer an invariant of motion. However, it is shown by simple calculations that the sets $\{\bar{x}=0\}$ and $\{\bar{\xi}=0\}$ still make invariant manifolds. The set $\{\bar{x}=0\}$ divides the future of the reaction due to its invariance (no trajectory can cross it). Here the transformation and the final form of 
the Hamiltonian are calculated by canonical perturbation theory $[28,29]$ as in the previous works [7-9,22-27]. The difference is the choice of terms to be held in the final Hamiltonian. In this Letter we call Eq. (5) "minimal normal form" (MNF) because it is a minimal functional form for obtaining the invariance of the manifolds.

To check the validity of the theory, we perform calculations by using a hydrogen atom in crossed electric and magnetic fields as a model system $[7,8]$,

$$
\begin{aligned}
H= & \frac{1}{2}\left(P_{X}^{2}+P_{Y}^{2}+P_{Z}^{2}\right)+\frac{1}{2}\left(X P_{Y}-Y P_{X}\right) \\
& -\frac{1}{R}+\frac{1}{8}\left(X^{2}+Y^{2}\right)-\mathcal{E} X,
\end{aligned}
$$

where $X, Y, Z$ are Cartesian coordinates, $P_{X}, P_{Y}, P_{Z}$ their conjugate momenta, and $R=\left(X^{2}+Y^{2}+Z^{2}\right)^{1 / 2}$. We use $\mathcal{E}=0.45$ for illustration of the present theory. In this system, there is a saddle point at $X=-\mathcal{E}^{-1 / 2}$ corresponding to ionization.

The validity of the normal form theory can be estimated by a microcanonical average of the residual error [30] in the Hamiltonian $\Delta H \stackrel{\text { def }}{=} H-\bar{H}$, which would be zero if the transformation were exact. In reality this is not exactly zero because the perturbational construction of the transformation contains truncation errors. Thus the approximation is better when the value of $|H-\bar{H}|$ is smaller. Figure 2 shows the microcanonical averages of the error in the Hamiltonian as functions of the energy. The normal form calculations are performed up to 8th order perturbation. The resulting expression of the final Hamiltonian $\bar{H}$ in MNF is available in an electronic database [31]. Phase space points on the surface $q_{1}=0$, where $q_{1}$ is the normal mode position coordinate along the reactive mode, were randomly sampled with the constraint $H=E$ and the root mean square of $\Delta H$ is taken. The constraint $q_{1}=0$ makes the sampling approximately "on the top of the barrier." As the total energy increases, the normal forms become worse due to higher nonlinearities, and at certain energy they even become worse than the harmonic approximation. Roughly, the larger error than the harmonic approximation means divergence of the series because the error increases as the order increases (the harmonic approximation is used as the zeroth order).

Figures 1(b) and 1(c) show the flow in the phase space by drawing some representative trajectories whose initial conditions were sampled randomly. Panel (b) depicts the trajectories at relatively low energy $E=0.05$ projected on the PNF coordinates. Since the PNF is a good description at this energy (see Fig. 2), the action $\bar{I}=\bar{x} \bar{\xi}$ is a good invariant of motion. Consequently the trajectories follow the hyperbolas given by constant action, without crossing with each other in the phase space. We can see a clear lamination in the picture of phase space flow. When the total energy increases, the phase space structure experiences a significant change. At $E=0.15$, the PNF is no longer a valid description, whereas the MNF still gives a good approximation to the true dynamics (see Fig. 2). Figure 1(c) shows the trajectories at this energy projected on the MNF coordinates. Since the action variable is not an invariant of motion in the MNF, the trajectories do not follow the hyperbolas, and crossings of trajectories with each other are also observed. One can no longer find a laminar flow in the phase space. However, no trajectories can cross the invariant manifolds $\{\bar{x}=0\}$ and $\{\bar{\xi}=0\}$ as expected from the theory. We can still predict the fate of the reaction by the sign of $\bar{x}$.

Next we take statistics of the "fate" of trajectories. Here, we sample 10000 initial conditions randomly on the surface $q_{1}=0$. When numerically propagated forward in time, the trajectory either goes out into the ionized region or is captured in the vicinity of the proton. Table I shows the number of the ionizing and the nonionizing trajectories with the sign of the initial values of $\bar{x}$. The numbers in the parentheses correspond to the "failures" of the theory in predicting the fate of the trajectory, compared to the number out of the parentheses showing the number of "successes." We can find significant improvements by MNF compared to the harmonic and PNF.

In summary, we have presented the analytical framework which enables us to assign and predict the fate of reaction even at a high energy regime. In Fig. 1(c), the chosen system, no recrossing was found over the surface $\bar{q}_{1}=0$. Note however that this is not a general consequence from Eq. (5), as we allow any functional forms of $g_{1}$ and $g_{2}$. What is exactly derived from the form of Eq. (5) is the invariance of $\{\bar{x}=0\}$ and $\{\bar{\xi}=0\}$, not the norecrossing property of $\left\{\bar{q}_{1}=0\right\}$. The significant point in the present theory is that we can still find an "impenetrable

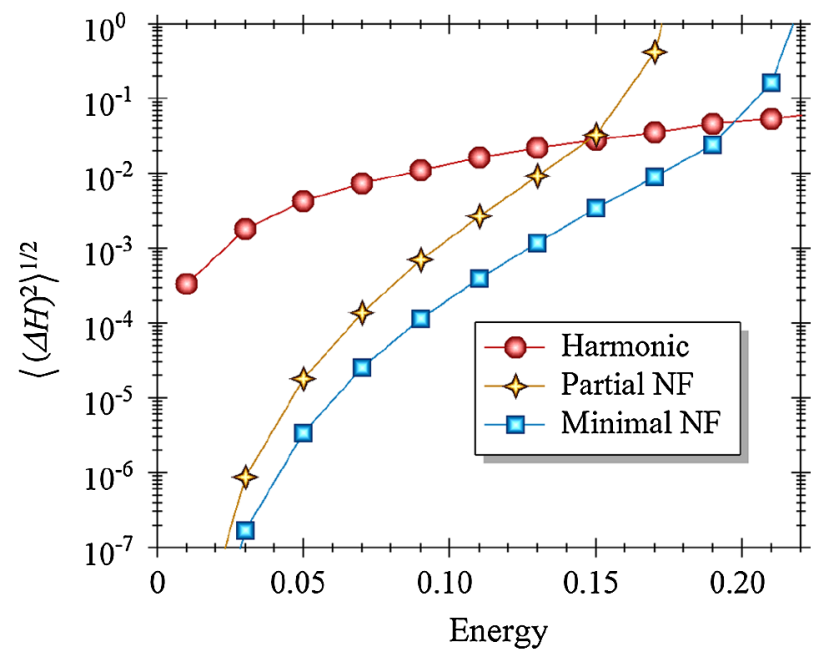

FIG. 2 (color online). Microcanonical averages of errors of normal form description calculated for a hydrogen atom in crossed electric and magnetic fields. The circle, plus, and square depict the error of harmonic approximation, partial normal form, and minimal normal form, respectively. 
TABLE I. The numbers of sampled trajectories against the sign of their initial values of the reaction coordinate $x$ and $\bar{x}$. Here $x$ is the reaction coordinate in harmonic approximation, and $\bar{x}$ those computed by either PNF or MNF.

\begin{tabular}{lcccccc}
\hline \hline \multirow{2}{*}{ Energy $^{\mathrm{a}}$} & \multicolumn{2}{c}{ Harmonic } & \multicolumn{2}{c}{ PNF } & \multicolumn{2}{c}{ MNF } \\
\hline 0.15 (ionizing) & 500 & $x<0$ & $\bar{x}>0$ & $\bar{x}<0$ & $\bar{x}>0$ & $(5)$ \\
0.15 (nonionizing) & $(2)$ & $(1743)$ & 6677 & $(93)$ & 6765 & $(3)$ \\
0.2 (ionizing) & 5025 & $(2026)$ & 6786 & $(265)$ & 7021 & $(30)$ \\
0.2 (nonionizing) & $(4)$ & 2945 & $(326)$ & 2623 & $(21)$ & 2928 \\
\hline \hline
\end{tabular}

${ }^{a}$ At each energy, the upper figures are the numbers of trajectories that are numerically found to reach the ionized region, while the lower figures are those captured in the well region, shown against the sign of the initial values of $x$ and $\bar{x}$. For example, out of the 10000 points randomly sampled on $q_{1}=0$ at $E=0.15,4971$ had $x<0$ ("predicted" to be nonionizing if the harmonic approximation is correct). Of the 4971 trajectories, 1743 were numerically found to be ionizing (although the sign of $x$ predicts them as nonionizing), while 3228 were nonionizing.

boundary" between reactive and nonreactive trajectories even when a no-recrossing TS may not exist. The crux was the relaxation of normalization in normal form theory, which yields a wider convergence radius of the theory in the region of rank-one saddles. By comparing the applicability ranges of the harmonic, partial, and minimal normal forms, we found a hierarchy of transitions as a function of the total energy of the system, from the regularly laminated flow to the chaotic one that is still divided into distinct regions by the impenetrable boundary of the reactivity. At yet higher energy, one may observe another transition, to fully chaotic reaction where even MNF cannot locate the invariant manifolds.

This work has been supported by the Japan Society for the Promotion of Science for Young Scientists (S. K.) and by JSPS, JST/CREST, Priority Area "Molecular Theory for Real Systems" (T. K.). The computations were partially performed using the Research Center for Computational Science, Okazaki, Japan.

*skawai@es.hokudai.ac.jp

[1] J. Zhang, D. Dai, C. C. Wang, S. A. Harich, X. Wang, X. Yang, M. Gustafsson, and R. T. Skodje, Phys. Rev. Lett. 96, 093201 (2006).

[2] W. Shiu, J. J. Lin, and K. Liu, Phys. Rev. Lett. 92, 103201 (2004).

[3] R. T. Skodje, D. Skouteris, D. E. Manolopoulos, S.-H. Lee, F. Dong, and K. Liu, Phys. Rev. Lett. 85, 1206 (2000).

[4] T. Bartsch, R. Hernandez, and T. Uzer, Phys. Rev. Lett. 95, 058301 (2005).

[5] T. Bartsch, T. Uzer, and R. Hernandez, J. Chem. Phys. 123, 204102 (2005).

[6] T. Bartsch, T. Uzer, J. M. Moix, and R. Hernandez, J. Chem. Phys. 124, 244310 (2006).

[7] S. Wiggins, L. Wiesenfeld, C. Jaffé, and T. Uzer, Phys. Rev. Lett. 86, 5478 (2001).

[8] T. Uzer, C. Jaffé, J. Palacián, P. Yanguas, and S. Wiggins, Nonlinearity 15, 957 (2002).
[9] T. Komatsuzaki and R. S. Berry, J. Chem. Phys. 110, 9160 (1999).

[10] C. Jaffe, S. D. Ross, M. W. Lo, J. Marsden, D. Farrelly, and T. Uzer, Phys. Rev. Lett. 89, 011101 (2002).

[11] C. P. Herrero and R. Ramírez, Phys. Rev. Lett. 99, 205504 (2007).

[12] M. Karplus, J. Phys. Chem. B 104, 11 (2000).

[13] S. S. Plotkin and P. G. Wolynes, Phys. Rev. Lett. 80, 5015 (1998).

[14] H. Eyring, J. Chem. Phys. 3, 107 (1935).

[15] E. Wigner, J. Chem. Phys. 5, 720 (1937).

[16] M. G. Evans and M. Polanyi, Trans. Faraday Soc. 31, 875 (1935).

[17] O. K. Rice and H. C. Ramsperger, J. Am. Chem. Soc. 50, 617 (1928).

[18] L. S. Kassel, J. Phys. Chem. 32, 1065 (1928).

[19] R. A. Marcus, J. Chem. Phys. 20, 359 (1952).

[20] J. C. Keck, Adv. Chem. Phys. 13, 85 (1967).

[21] D. G. Truhlar and B. C. Garrett, Acc. Chem. Res. 13, 440 (1980).

[22] Geometrical Structures of Phase Space in Multidimensional Chaos: Applications to Chemical Reaction Dynamics in Complex Systems, Advances in Chemical Physics Vols. 130A and 130B, edited by M. Toda, T. Komatsuzaki, T. Konishi, R. S. Berry, and S. A. Rice (Wiley, New York, 2005), and references therein.

[23] S. Kawai, H. Teramoto, C.-B. Li, T. Komatsuzaki, and M. Toda, Adv. Chem. Phys. (to be published).

[24] T. Komatsuzaki and R. S. Berry, Proc. Natl. Acad. Sci. U.S.A. 98, 7666 (2001).

[25] T. Komatsuzaki and R. S. Berry, Adv. Chem. Phys. 123, 79 (2002).

[26] R. Hernandez, J. Chem. Phys. 101, 9534 (1994).

[27] C.-B. Li, A. Shoujiguchi, M. Toda, and T. Komatsuzaki, Phys. Rev. Lett. 97, 028302 (2006).

[28] A. Deprit, Celest. Mech. 1, 12 (1969).

[29] A. Lichtenberg and M. Lieberman, Regular and Chaotic Dynamics (Springer, New York, 1992).

[30] S. Kawai, C. Jaffé, and T. Uzer, J. Phys. B 38, S261 (2005).

[31] See supplementary material at http://link.aps.org/ supplemental/10.1103/PhysRevLett.105.048304 for the full explicit expressions of the MNF Hamiltonian. 